\title{
Macrophage Activation Syndrome: different mechanisms leading to a one clinical syndrome
}

\author{
Claudia Bracaglia* ${ }^{*}$, Giusi Prencipe and Fabrizio De Benedetti
}

\begin{abstract}
Background: Macrophage activation syndrome (MAS) is a severe complication of rheumatic disease in childhood, particularly in systemic Juvenile Idiopathic Arthritis (sJIA). It is characterize by an uncontrolled activation and proliferation of T lymphocytes and macrophages.

Main content: MAS is currently classified among the secondary or acquired forms of haemophagocytic lymphohistiocytosis $(\mathrm{sHLH})$. The reason is that MAS shares clinical and laboratory features with primary genetic $\mathrm{HLH}(\mathrm{pHLH})$. In this context is conceivable that some of the pathogenic mechanisms of pHLH may be involved in other forms of HLH. Heterozygosity for mutations of genes involved in pHLH may lead to a cytotoxic defect and to a development of clinical overt disease. But other different contributors might be involved to the development of MAS such as infections or underlying inflammation. In MAS, the inflammatory status of the patient is a major contributor of the disease. Indeed, the majority of the MAS episodes occurs during active disease phases or at disease onset. In addition, recent evidence in animals and humans suggest that genetics may also play a major role in contributing to hyperinflammation and particularly to macrophages hyper-responses.
\end{abstract}

Conclusions: We hypothesize that HLH may be one unique clinical syndrome, to whose generation different mechanisms may contribute, and maintained by one final effector mechanism.

Keywords: Macrophage activation syndrome, Haemophagocytic lymphohistiocytosis, IL-6, IL-18, IFNץ, Inflammasome, Natural killer

\section{Background}

The term Macrophage Activation Syndrome (MAS) identifies a potentially fatal complication of rheumatic diseases. It occurs usually in the context of systemic Juvenile Idiopathic Arthritis (sJIA), but it may occur also, albeit more rarely, in systemic Lupus Erythematosus and Kawasaki disease.

MAS is typically characterized by high fever, lymphoadenopathy, hepatosplenomegaly variably associated with hemorrhages, signs of liver, central nervous system and kidney involvement, and may lead to multiple organ failure. Laboratory abnormalities include decrease in white blood cells, platelet and hemoglobin,

* Correspondence: claudia.bracaglia@opbg.net

Division of Rheumatology, Ospedale Pediatrico Bambino Gesù IRCCS, Piazza S. Onofrio 4, 00165 Rome, Italy hypertransaminasemia, marked increase in ferritin, and evidence for intravascular coagulation activation.

\section{MAS clinical and laboratory features}

Minoia et al. collected in a multinational multicentre study 362 cases of patients with diagnosis of MAS, in the context of sJIA, established by the treating physicians. All patients had fever, a relevant percentage of them (58\%) had splenomegaly and lymphadenopathy (51\%). Surprisingly, $35 \%$ of them had symptoms suggestive of CNS involvement, $16 \%$ had pericarditis, which is consistent with systemic JIA, and $20 \%$ had clinical evidence of consumption coagulopathy. See Appendix 1: Case 1 for laboratory abnormalities in sJIA and MAS. In the large multinational study, the great majority of the patients had high ferritin. All the patients had liver involvement and, in the great majority of them, transaminases (94\%) and LDH (94\%) were abnormal. Anaemia 
(92\%) was very frequent; however, thrombocytopenia (70.3\%) and neutropenia (54.5\%) were not as frequent as in primary HLH (pHLH). Triglycerides (93\%) were elevated in the majority of patients; evidence of intravascular coagulation, as demonstrated by elevated d-dimer, was present in the great majority of the patients (97\%). Fibrinogen was not below the normal limit in a significant portion of patients (48.6\%), as expected in highly inflammatory disease where fibrinogen behaves as a positive acute phase protein [1].

\section{Classification}

In general, MAS is classified among the secondary forms of haemophagocytic lymphohistiocytosis (sHLH), as a sHLH occurring in the context of a rheumatic disease (Table 1), the reason being that HLH and MAS share a number of clinical and laboratory features. The most significant differences are due to the inflammatory background of MAS: particularly platelet counts, as well as fibrinogen levels, are usually higher in MAS compared to pHLH. Primary HLH is caused by mutation in genes that code for proteins that are involved in "perforin-mediated" cytolytic function. They are either proteins such as perforin, directly involved in cytotoxicity, or proteins

Table 1 Classification of primary (genetic) and secondary (reactive) HLH [46-48]

\begin{tabular}{|c|c|c|}
\hline & GENE & FUNCTION \\
\hline \multicolumn{3}{|l|}{ Genetic HLH } \\
\hline \multicolumn{3}{|l|}{ Primary or Familial HLH (pHL) } \\
\hline $\mathrm{pHL}-1$ & Unknown & \\
\hline $\mathrm{pHL}-2$ & PRF1 & Pore-forming protein \\
\hline $\mathrm{pHL}-3$ & UNC13D & $\begin{array}{l}\text { Vesicle priming (impair } \\
\text { granule exocytosis) }\end{array}$ \\
\hline $\mathrm{pHL}-4$ & STX11 & $\begin{array}{l}\text { Vesicle transport } \\
\text { and fusion }\end{array}$ \\
\hline $\mathrm{pHL}-5$ & STXBP2 & $\begin{array}{l}\text { Vesicle transport } \\
\text { and fusion }\end{array}$ \\
\hline \multicolumn{3}{|l|}{$\begin{array}{l}\text { Sporadic associated } \\
\text { with Immunodeficiencies }\end{array}$} \\
\hline $\mathrm{CHS}$ & LYST & Vesicle transport \\
\hline GS-2 & RAB27A & Vesicle docking \\
\hline$X L P-1 / X L P-2$ & $\begin{array}{l}\text { SH2DIA } \\
\text { BIRC4 }\end{array}$ & $\begin{array}{l}\text { Signal transduction } \\
\text { and activation } \\
\text { of lymphocytes }\end{array}$ \\
\hline \multicolumn{3}{|l|}{$\begin{array}{l}\text { Secondary HLH or Acquired } \\
\text { HLH or Reactive HLH }\end{array}$} \\
\hline \multicolumn{3}{|l|}{$\begin{array}{l}\text { Infections (EBV, } \\
\text { Leishmania, H1N1 ...) }\end{array}$} \\
\hline \multicolumn{3}{|l|}{$\begin{array}{l}\text { Rheumatic diseases (sJIA...) } \\
\rightarrow \text { MAS or rheuma-HLH }\end{array}$} \\
\hline $\begin{array}{l}\text { Malignancies } \\
\text { (lymphoma...) }\end{array}$ & & \\
\hline
\end{tabular}

involved in vesicle transport and fusion with the plasma membrane.

There are several conditions associated with secondary or acquired or reactive HLH. Although definitive epidemiologic data are lacking, sHLH appear to be much more frequent than pHLH. Among infection associated HLH, Epstein Barr Virus is certainly the most common trigger [2]. Additional typical triggers include other Herpes viruses, Leishmania and H1N1 influenz [3-5]. There are a number of cases associated with lymphoma and leukaemia, most frequently $\mathrm{T}$ cell receptor gammadelta bearing $\mathrm{T}$ cell lymphomas. These appear to be rare in children, but this occurrence is rather frequent in adults [6]. HLH can also be associated to an underlying rheumatic disease with sJIA being by far the most frequent one. This form of sHLH is what paediatric rheumatologist call MAS or rheuma-HLH. There is a significant number of cases in which an apparent underlying disease or an apparent underlying infection cannot be found [7].

\section{Genetics of MAS: contribution to defective cytotoxicity}

As mentioned above, in pHLH, all causative genes code for proteins involved in the cytotoxic pathway, in which cytolytic granules are transported to and fused with the cell membrane, and eventually pore-forming proteins, such as perforin, are secreted in these immunological synapses $[8,9]$. If HLH is considered one clinical syndrome is conceivable that some of the pathogenic mechanisms of pHLH may be involved in other forms of HLH. See Appendix 2: Case 2 for potential impact of heterozygosity for mutations of pHLH genes on development of clinical overt disease and of a defective cytotoxicity [10].

One may also expect that a significant role for genetics may impact on the recurrence of the disease. Indeed, recurrent MAS in patients with sJIA is a well-known phenomenon. However, information on the frequency of recurrences is lacking from the large series reported in recent papers [1, 11-14].

Kaufman et al. performed whole exome sequencing in 14 MAS cases on sJIA and found that 5 out of 14 patients (36\%) were heterozygous for at least one mutation of the known genes related to pHLH. In addition, they found that 22 rare variants occurred in at least two patients. Most of these variants code for proteins involved in microtubule organization and vesicle transport [11]. This suggested that cellular assembly and cytoscheletal organization may be involved in the potential defect in cellular cytotoxicity of these patients. A number of other studies have previously looked at the frequency of variants in the genes causing pHLH and overall they all found a number of patients carrying either hypomorphic variants or pathogenic mutations in heterozygosity in 
both UNC13d [15] and PRF1 [13]. In the context of the Italian National Registry of familial HLH we performed mutation analysis of genes involved in pHLH in 31 patients with sJIA and MAS. The analysis included PRF1, UNC13d, STX11, STXBP2and RAB27a, and identified monoallelic mutations in 11 of the 31 patients (35.5\%). Three out of 31 patients (9.7\%) carried a mutation in 2 genes. Interestingly, it has been recently reported that mice carrying heterozygous mutation in more than one gene involved in pHLH carry a significant higher risk to develop HLH following viral infection [16]. Overall, clinical and laboratory feature of MAS in patients carrying the mutations were not different from those of patients who do not carry the mutation. However, recurrences of MAS seem to be more frequent in patients who carry the mutations (mutated 27\% versus non-mutated 10\%). In our series of patients one patient died because of MAS and she was heterozygous for a known pathogenic PRF1 mutation (N252S) [17]. Altogether these observations, far from being conclusive, point to the presence of a genetic contribution to MAS similar to that of pHLH. All together these observations suggest that events related to the pathogenesis of pHLH are relevant in sHLH in general, and in MAS in particular: a) accumulation of partial genetic defect in one, or more than one, of the known pHLH related gene b) the possible dominant negative effects of some heterozygous variants $[10,18]$. Defective cytotoxicity may impair clearance of infected cells, and/or prolong lymphocyte-APC interactions, both events leading to increased pro-inflammatory cytokine production (e.g. IFN $\gamma$ ) by $\mathrm{T}$ lymphocytes $[10,19]$.

\section{Different contributors to the development of a unique clinical syndrome}

In $\mathrm{pHLH}$ the contribution of genetics is so strong that, even in the absence of any demonstrable trigger, genetically caused immune abnormalities are sufficient to drive the individual in full blown clinical HLH. In HLH secondary to infection, in some instances, the strength of the infectious agent is sufficient to cause HLH. Most of the patients who died of H1N1 or H5N1 died with HLH [20]. There are other agents that cause HLH rather often, as mentioned above. In these instances, there must be a contribution from the infectious agent, or more precisely from the response elicited by a given infectious agent. In this respect, it is interesting to note that gene expression profiles of peripheral blood mononuclear cells from patients with symptomatic mononucleosis show evident similarities with gene expression profiles of patients with HLH or MAS [21]. However, there must be also a contribution from the genetic background. Schulert et al. found rare variants in pHLH related genes in 5 out 14 cases of fatal H1N1 influenza infection, all of whom had evidence of hemophagocytosis. Five patients were heterozygous for LYST mutation, two of them carried also a PRF1 mutation [22].

In MAS, the HLH clinical syndrome develops on the background of a highly inflammatory disease such as sJIA. Also in sJIA an infectious trigger appears to play a role. Several MAS appears to be triggered by acute infections in patients with active sJIA. In a recent large multinational survey, this occurrence was documented in approximately $1 / 3$ of the cases [1]. In MAS, it is obvious that an additional contribution must be provided by the inflammatory status of the patient; indeed, the majority of the MAS episodes occurs during active disease phases or at disease onset.

\section{Contribution of active inflammation to the development of MAS}

We generated a model of MAS using the interleukin-6 (IL-6) transgenic mouse (IL-6TG). In these mice, administration of Toll-like receptor (TLR) ligands, including lipopolysaccharides (LPS) and poly(I:C), induces the development of MAS by mimicking an acute infection on a background of high levels of IL-6 [23]. This experimental approach recapitulates what occurs in patients with systemic JIA: an infection triggers MAS on a background of active disease, which is indeed characterized by high levels of IL-6.

Compared to wild type mice, LPS-challenged IL-6 transgenic mice display an increased fatality rate and show hematologic and biochemical features typically present in MAS patients, including lower neutrophils and platelet count, higher levels of ferritin and LDH, as well as a cytokine storm.

When we looked at the stimulatory effect of IL-6, we showed that, in vitro, prolonged exposure of human macrophages to IL-6 leads to increased production of cytokines and chemokines, including Tumor Necrosis Factor- $\alpha$ (TNF- $\alpha$ ) and (C-X-C Motif) Ligand 8 (CXCL- 8 ), both at a basal level and upon stimulation with TLR ligands, suggesting a role for IL-6 in amplifying the inflammatory response [24]. In addition, we demonstrated that IL-6 amplifies TLR-induced inflammatory response also in cells originating from inflammatory site, such as synovial fluid mononuclear cells and fibroblast-like synoviocytes isolated from arthritides patients. Indeed, treatments of these cells with IL-6 increased basal production of CXCL8, C-C motif chemokine ligand 2 (CCL2) and Interleukin-1 $\beta$ (IL-1 $\beta$ ) following poly(I:C) and muramyl dipeptide stimulation [24].

The other major feature of MAS in the context of sJIA is the transitory defect in cellular cytotoxic function. Grom et al. demonstrated that most of the sJIA patients have profoundly decreased Natural Killer (NK) cell activity [25]. The reduced NK cytotoxic activity observed was 
associated, in some patients, to a decreased perforin expression on NK cells and, in other patients, to a decreased perforin expression on T-cells. However, overall, the great majority of patients display a NK cytotoxic activity defect.

Consistently, we found that NK activity was impaired also in the murine model of MAS, the IL-6TG. Indeed, IL6TG mice challenged with poly(I:C), a substitute for viral dsRNA, display a significant decrease in NK cell cytotoxicity, associated to lower expression levels of perforin and granzyme B, in the absence of altered granule exocytosis [26]. An example of IL-6 leading to decreased cytolytic activity of lymphocytes on top of an underlying genetic defect has been shown [10]. Similarly, in vitro experiments revealed that exposure of human PBMCs to high levels of IL-6, such as those typically present in sJIA, inhibits NK cell cytotoxicity by down-regulating the expression of perforin and granzyme B. Altogether, this evidence suggests that high circulating levels of IL-6 in JIA patients, besides their role in increasing macrophage activation and TLR response, play a major role in inducing a transitory defect in cytotoxicity on top of a subclinical genetic defect.

In addition to IL-6, other inflammatory cytokines may play a role in predisposition to MAS. Indeed, even in patients treated with tocilizumab, in which IL-6 was fully neutralized, MAS may occur [27]. One of the potential candidates is IL- $1 \beta$. There is a number of observations pointing to the efficacy of high dose of anakinra in at least some patients with MAS [28, 29]. On the other hand, there is a number of studies describing patients who developed clinically overt MAS while being treated with both IL-1 blockers anakinra and canakinumab [30-32]. In apparent contrast, in criopyrinopathies, which are the purely IL- $1 \beta$ mediated, cases of MAS are not described. Overall, these data would suggest that neither IL-6 nor IL-1 $\beta$ alone may be the major contributors to the predisposition to MAS.

Increasing number of evidence suggests that interleukin18 (IL-18) might be another relevant mediator in sJIA. In a study performed in Japan, the subset of sJIA patients characterized by high levels of IL-18 (and conversely low levels of IL-6) had a higher frequency of MAS episodes [12]. These data need to be confirmed in a Western population. Albeit in a small number of patients, data from Put et al. are consistent with this observation: in sJIA patients high IL-18 levels do not markedly increase during active MAS, pointing to IL-18 being a predisposing cytokine [33]. The mechanisms behind the predisposing effect of IL-18 are not clear yet. It is well know that IL-18 affects NK cell activity, but preliminary evidence suggests that it may also regulate macrophage responses [34].

\section{Contribution of genetics to hyperinflammation}

In addition to the known role of genetics of cytotoxicity genes in HLH, recent evidence in animals and humans suggest that genetics may also play a major role in contributing to hyperinflammation and particularly to macrophages hyper-responses. Indeed, data in the animals clearly support this hypothesis. The most studied model of genetically driven HLH are perforin knock-out mice which, upon LCMV infection, develop full blown HLH and die [35]. When UNC13d deficient mice are crossed with mice deficient for MYD88, which is a central element in TLR intracellular signalling, the animals are protected from disease development, demonstrating that, at least in animals, TLR signalling and macrophage responses are crucial [36]. Indirect evidence in humans has been provided by a study suggesting that a polymorphism in IRF5, a transducer of intracellular TLR, is associated with the risk of MAS in patients with sJIA [37]. In addition and much more importantly, direct evidence in humans is provided by the recent observation that gain of function mutations of NLRC4, an inflammasome sensor, cause a disease characterized by recurrent or dramatic HLH. This observation demonstrates in humans that genetically induced abnormalities in autoinflammation cause HLH. The disease, still described in very few patients appear to be characterized by early onset of fever, skin rash, severe gastro-intestinal involvement, splenomegaly and recurrent, severe MAS/HLH [38, 39]. Immunological studies revealed that the most striking abnormality is represented by surprisingly high levels of IL-18 pointing to the fact that the NLRC4 protein is particularly important in regulating IL-18 production and further supporting the role of IL-18 as a predisposing factor to HLH/MAS [38].

\section{Different contributors and one pathogenic mediator?}

It is reasonable to hypothesize that several factors, acting at different steps, contribute to the development of MAS and sHLH. A multilayer model of pathogenic events leading to the development of MAS has been proposed, including genetics, inflammatory activity and infection, contributing to reaching a threshold of activation that results in overt MAS [40]. In perforin knock-out mice infected with LCMV, the most used mouse model of primary $\mathrm{HLH}$, neutralization of a number of cytokines does not produce any relevant clinical effect. However, neutralization of IFNY leads to protection from disease and to survival of the animals [35]. Based on a number of experiments both in mice and on human cells, it has been hypothesized that mutations of genes impacting on cytotoxic activity generate an inability of cytotoxic $\mathrm{T}$ cells to lyse the target cells. This defect leads to prolonged hyperactivation of $\mathrm{T}$ cells with hyperproduction of $\operatorname{IFN} \gamma[10,19]$. High levels of IFN $\gamma$ leads to activation of macrophages, as a compensatory mechanism aimed at phagocytosis of 
the infected cells. Indeed, these patients do not succumb from overwhelming infections. These activated macrophages produce very large amounts of inflammatory cytokines and generate a cytokine storm, which is the driver of the clinical and laboratory features of HLH and eventually leads the host to death, if left untreated.

Translating from $\mathrm{pHLH}$ to MAS and based on the similarities in clinical and laboratory features as well as in genetic contribution, we have recently investigated whether IFN $\gamma$ could potentially be involved also in MAS. IFN $\gamma$ levels were found to be higher in patients with active MAS at sampling compared to patients with active sIIA without MAS at sampling [41]. Using the above mentioned mouse model of MAS in IL-6 transgenic mice we have also found that neutralization of IFN $\gamma$ improved survival and reverted biochemical abnormalities (Prencipe G. 2016 submitted). Interestingly, in patients with MAS a marked increase in CXCL9 levels was also found. CXCL9 is known to be specifically induced by IFN $\gamma$ [42]. Indeed in patients with active MAS levels of IFN $\gamma$ and CXCL9 were strictly related to laboratory features of MAS, including ferritin levels, white blood cell and platelet counts, and LDH [41]. These correlations were not present in patients with active sJIA without MAS. These data suggest that during MAS, but not during active sJIA, there is a specific activation of IFNY and of the IFN $\gamma$ pathway. Noteworthy in the NLRC4 patient, reported by Canna et al., one of the cytokines that increases significantly during active HLH episodes is indeed CXCL9 [38]. We also found high levels of CXCL9 in a patient with NLRC4 induced disease (unpublished). Observations in MAS and in NLRC4-induced disease show that hyperinflammation induced by dysregulated inflammatory responses may directly cause overproduction of IFNY.

\section{Conclusion}

All together these observations are consistent with the hypothesis that HLH may be one clinical syndrome maintained by one effector mechanism, which involves increased macrophage activation and increased T-cells activation. In this loop, IFNY seems to play a major role. In classical pHLH, genetic mutations and the consequent defect in cytotoxic pathways are sufficient to drive a patient above the threshold eliciting this vicious loop. NLRC4-induced disease provides proof of concept that genetic predisposition to inflammasome hyper-response alone can drive patients directly into the same vicious loop. In patients with MAS an additive/synergist role is played by several contributors, including infectious triggers, genetics, of both cytotoxicity and inflammatory response, and inflammatory activity of the underlying rheumatic disease. Recently generated evidence points to IFNY as the major mediator also of HLH secondary to infections [43, 44], as well as of MAS. These findings may have significant translational value. A monoclonal antibody to IFN $\gamma$ is in clinical trial in primary HLH with initial satisfactory response [45] and a trial in MAS is being planned.

\section{Appendix 1: Case 1 \\ Case 1}

An 8 years old Caucasian boy with sJIA onset one year before, suddenly developed persistent fever, generalised seizures, hypotension, purpura and splenomegaly. Laboratory picture was completely different if compared to his laboratory at sJIA onset. Blood count showed leukopenia with neutrophenia and anemia. Erithrocyte sedimentation rate (ESR) was relatively low, however CRP was high as it was. Fibrinogen was low because of intravascular activation of the coagulation, d-dimers were high. He presented also liver involvement with some degree of liver insufficiency. The most striking feature was hyperferritinimia, which was markedly higher compared to sJIA presentation (Table 2). This case represents a typical picture of MAS complicating sJIA. Changes in laboratory features are the hallmark and these have been recently mathematically characterized in multination effort [1]. However, MAS may represents a significant challenge when occurring at sJIA onset.

Table 2 Patient's laboratory features

\begin{tabular}{lcc}
\hline Laboratory Features & SJlA presentation & MAS presentation \\
\hline White blood cells, $\times 10^{9} / \mathrm{L}$ & 15.04 & 3.02 \\
Neutrophils \% & $86 \%$ & $19 \%$ \\
Haemoglobin, g/dL & 9.08 & 7.09 \\
Platelet, $\times 109 / \mathrm{L}$ & 640 & 125 \\
Ferritin, $\mathrm{ng} / \mathrm{mL}$ & 880 & 11345 \\
ESR, $\mathrm{mm} / \mathrm{h}$ & 98 & 32 \\
CRP, $\mathrm{mg} / \mathrm{dL}$ & 13.08 & 19.07 \\
Fibrinogen, $\mathrm{mg} / \mathrm{dL}$ & 560 & 88 \\
D-dimers, $\mathrm{ng} / \mathrm{mL}$ & $<10$ & $>40$ \\
Sodium, $\mathrm{mEq} / \mathrm{L}$ & 136 & 129 \\
ALT, U/L & 23 & 180 \\
AST, U/L & 18 & 145 \\
LDH, U/L & 380 & 1910 \\
Bilirubin, mg/dL & 0.06 & 2.06 \\
\hline
\end{tabular}

ESR, erythrocyte sedimentation rate; CRP, C-reactive protein; $A L T$, alanine aminotransferase; AST, aspartate aminotransferase; LDH, lactate dehydrogenase 


\section{Appendix 2: Case 2}

\section{Case 2}

A 15 years-old, Caucasian male presented with persistent fever since 4 weeks, diarrhoea vomiting, skin rash, hepatosplenomegaly, and elbows arthritis. Blood tests showed high ferritin (5896 ng/ml), liver inflammation (AST 224 IU/l, ALT 635 IU/l, LDH 676 IU/l), and intravascular activation of coagulation with high d-dimers $(2.26 \mu \mathrm{g} / \mathrm{ml})$, and a bone marrow biopsy showed hemophagocytosis. He was treated with glucocorticoids, cyclosporine A and cyclophosphamide with good response. Three years later, he presented again with persistent fever, skin rash, diffuse arthralgia, and myalgia. He referred to the malaise and arthralgia as being the "same disease" as the one 3 years before. As soon as ferritin started to rise significantly $(5481 \mathrm{ng} / \mathrm{ml})$, dexamethasone and cyclosporine A were started with a prompt response. He was found to carry a single copy heterozygous missense mutation in RAB27a. This same mutation was found in his father, who was apparently healthy, but who had a baseline moderately high serum ferritin level (800 ng/ml). Functional tests showed a marginal defect in CD107a degranulation in the patient and his healthy father [10]. Indeed experiments with this very mutation have shown an impact on cytotoxic function as well as on the duration of the immune synapses [10]. This patient appears to have had two episodes of HLH, which cannot be strictly defined as primary or genetic HLH; it is however, tempting to speculate that carriage of this mutation plays an important role in the two recurrences of secondary HLH.

\section{Abbreviations}

CCL2: C-C motif chemokine ligand 2; CXCL8: (C-X-C Motif) Ligand 8; CXCL9: (C-X-C Motif) Ligand 9; HLH: Haemophagocytic lymphohistiocytosis; IL-18: Interleukin-18; IL-1ß: Interleukin-1ß; IL-6: Interleukin-6; IL-6TG: IL-6 transgenic mouse; IRF5: Interferon regulatory factor 5; LCMV: Lymphocytic choriomeningitis virus; LPS: Lipopolysaccharides; MAS: Macrophage activation syndrome; MYD88: Myeloid differentiation primary response 88; NK: Natural killer; PBMC: Peripheral blood mononuclear cell; pHLH: Primary or familial haemophagocytic; lymphohistiocytosis; sHLH: Secondary or acquired haemophagocytic lymphohistiocytosis; sJIA: Systemic juvenile idiopathic arthritis; TLR: Toll-like receptor; TNF-a: Tumor necrosis factor-a

\section{Acknowledgements}

Not applicable.

\section{Funding}

Giusi Prencipe was supported in her research by the Italian Ministry of Health (Rome, Italy) Young Investigator Grants "GR-2011-02347874".

\section{Availability of data and materials}

Data presented in the review are available in the references listed below.

\section{Authors' contributions}

$\mathrm{CB}$ conducted the literature review and wrote the manuscript; GP wrote the section on mouse model; FDB reviewed the manuscript. All authors read and approved the final manuscript.

\section{Competing interests}

Claudia Bracaglia and Giusi Prencipe declare that they have no competing interests. Fabrizio De Benedetti declares competing interests with Novartis, Novimmune, Hoffmann-La Roche, SOBI, AbbVie.

\section{Consent for publication}

A consent for publication was collected from the patients and their parents described in Appendix 1: Case 1 and Appendix 2: Case 2.

\section{Ethics approval and consent to participate}

The Ethical Committee of IRCCS Ospedale Pediatrico Bambino Gesù, Study number 446/2011, Protocol number 494_11 approved the study [38].

A written consent to participate to the study was collected for all participants. A consent to publish individual patient data is included in the written consent obtained from legal parent or guardian.

Received: 3 September 2016 Accepted: 27 December 2016

Published online: 17 January 2017

\section{References}

1. Minoia F, Davi S, Horne A, Demirkaya E, Bovis F, Li C, et al. Clinical features, treatment, and outcome of macrophage activation syndrome complicating systemic juvenile idiopathic arthritis: a multinational, multicenter study of 362 patients. Arthritis Rheumatol. 2014;66(11):3160-9.

2. Hashemi-Sadraei N, Vejpongsa P, Baljevic M, Chen L, Idowu M. Epstein-Barr virus-related hemophagocytic lymphohistiocytosis: hematologic emergency in the critical care setting. Case Rep Hematol. 2015;2015:491567. Pubmed Central PMCID: 4338404

3. Kilani B, Ammari L, Kanoun F, Ben Chaabane T, Abdellatif S, Chaker E. Hemophagocytic syndrome associated with visceral leishmaniasis. Int J Infect Dis. 2006;10(1):85-6.

4. Ozdemir $H$, Ciftci E, Ince EU, Ertem M, Ince E, Dogru U. Hemophagocytic lymphohistiocytosis associated with 2009 pandemic influenza A (H1N1) virus infection. J Pediatr Hematol Oncol. 2011;33(2):135-7.

5. Singh G, Shabani-Rad MT, Vanderkooi OG, Vayalumkal JV, Kuhn SM, Guilcher GM, et al. Leishmania in HLH: a rare finding with significant treatment implications. J Pediatr Hematol Oncol. 2013;35(3):e127-9.

6. Lehmberg K, Sprekels B, Nichols KE, Woessmann W, Muller I, Suttorp M, et al. Malignancy-associated haemophagocytic lymphohistiocytosis in children and adolescents. Br J Haematol. 2015;170(4):539-49.

7. Xu XJ, Tang YM, Song H, Yang SL, Xu WQ, Zhao N, et al. Diagnostic accuracy of a specific cytokine pattern in hemophagocytic lymphohistiocytosis in children. J Pediatr. 2012;160(6):984-90 e1.

8. Chandrakasan S, Filipovich AH. Hemophagocytic lymphohistiocytosis: advances in pathophysiology, diagnosis, and treatment. J Pediatr. 2013; 163(5):1253-9.

9. Stepp SE, Dufourcq-Lagelouse R, Le Deist F, Bhawan S, Certain S, Mathew PA, et al. Perforin gene defects in familial hemophagocytic lymphohistiocytosis. Science. 1999;286(5446):1957-9.

10. Zhang M, Bracaglia C, Prencipe G, Bemrich-Stolz CJ, Beukelman T, Dimmitt RA, et al. A heterozygous RAB27A mutation associated with delayed cytolytic granule polarization and hemophagocytic lymphohistiocytosis. J Immunol. 2016;196(6):2492-503. Pubmed Central PMCID: PMC4779709.

11. Kaufman KM, Linghu B, Szustakowski JD, Husami A, Yang F, Zhang K, et al. Whole-exome sequencing reveals overlap between macrophage activation syndrome in systemic juvenile idiopathic arthritis and familial hemophagocytic lymphohistiocytosis. Arthritis Rheumatol. 2014;66(12): 3486-95. Pubmed Central PMCID: 4321811.

12. Shimizu M, Nakagishi $Y$, Inoue N, Mizuta M, Ko G, Saikawa $Y$, et al. Interleukin-18 for predicting the development of macrophage activation syndrome in systemic juvenile idiopathic arthritis. Clin Immunol. 2015; 160(2):277-81.

13. Vastert SJ, van Wijk R, D'Urbano LE, de Vooght KM, de Jager W, Ravelli A, et al. Mutations in the perforin gene can be linked to macrophage activation syndrome in patients with systemic onset juvenile idiopathic arthritis. Rheumatology (Oxford). 2010;49(3):441-9.

14. Zhang K, Biroschak J, Glass DN, Thompson SD, Finkel T, Passo MH, et al. Macrophage activation syndrome in patients with systemic juvenile idiopathic arthritis is associated with MUNC13-4 polymorphisms. Arthritis Rheum. 2008;58(9):2892-6. Pubmed Central PMCID: 2779064. 
15. Hazen MM, Woodward AL, Hofmann I, Degar BA, Grom A, Filipovich AH, et al. Mutations of the hemophagocytic lymphohistiocytosis-associated gene UNC13D in a patient with systemic juvenile idiopathic arthritis. Arthritis Rheum. 2008:58(2):567-70.

16. Sepulveda FE, Garrigue A, Maschalidi S, Garfa-Traore M, Menasche G, Fischer A, et al. Polygenic mutations in the cytotoxicity pathway increase susceptibility to develop HLH immunopathology in mice. Blood. 2016;127(17):2113-21.

17. Bracaglia C, Sieni E, Da Ros M, De Fusco C, Micalizzi C, Cetica V, Ciambotti B, Coniglio ML, Insalaco A, De Benedetti F, Aricò M. Mutations of familial hemophagocytic lymphohistiocytosis (FHL) related genes and abnormalities of cytotoxicity function tests in patients with macrophage activation syndrome (MAS) occurring in systemic juvenile idiopathic arthritis (sIIA). Pediatr Rheumatol Online J. 2014;12 Suppl 1:53. Pubmed Central PMCID: PMC4184267. Epub Published online 2014 Sep 17.

18. Spessott WA, Sanmillan ML, McCormick ME, Patel N, Villanueva J, Zhang K, et al. Hemophagocytic lymphohistiocytosis caused by dominant-negative mutations in STXBP2 that inhibit SNARE-mediated membrane fusion. Blood. 2015;125(10):1566-77. Pubmed Central PMCID: 4351505.

19. Jenkins MR, Rudd-Schmidt JA, Lopez JA, Ramsbottom KM, Mannering SI, Andrews DM, et al. Failed CTL/NK cell killing and cytokine hypersecretion are directly linked through prolonged synapse time. J Exp Med. 2015;212(3): 307-17. Pubmed Central PMCID: 4354371.

20. Shrestha B, Omran A, Rong P, Wang W. Report of a fatal pediatric case of hemophagocytic lymphohistiocytosis associated with pandemic influenza a (H1N1) infection in 2009. Pediatr Neonatol. 2015;56(3):189-92.

21. Dunmire SK, Odumade OA, Porter JL, Reyes-Genere J, Schmeling DO, Bilgic $\mathrm{H}$, et al. Primary EBV infection induces an expression profile distinct from other viruses but similar to hemophagocytic syndromes. PLoS One. 2014;9(1):e85422. Pubmed Central PMCID: 3894977.

22. Schulert GS, Zhang M, Fall N, Husami A, Kissell D, Hanosh A, et al. Wholeexome sequencing reveals mutations in genes linked to hemophagocytic lymphohistiocytosis and macrophage activation syndrome in fatal cases of H1N1 influenza. J Infect Dis. 2016;213(7):1180-8.

23. Strippoli R, Carvello F, Scianaro R, De Pasquale L, Vivarelli M, Petrini S, et al. Amplification of the response to toll-like receptor ligands by prolonged exposure to interleukin-6 in mice: implication for the pathogenesis of macrophage activation syndrome. Arthritis Rheum. 2012;64(5):1680-8.

24. Caiello I, Minnone G, Holzinger D, Vogl T, Prencipe G, Manzo A, et al. IL-6 amplifies TLR mediated cytokine and chemokine production: implications for the pathogenesis of rheumatic inflammatory diseases. PLoS One. 2014; 9(10):e107886. Pubmed Central PMCID: PMC4182736.

25. Grom AA, Villanueva J, Lee S, Goldmuntz EA, Passo MH, Filipovich A Natural killer cell dysfunction in patients with systemic-onset juvenile rheumatoid arthritis and macrophage activation syndrome. J Pediatr. 2003;142(3):292-6.

26. Cifaldi L, Prencipe G, Caiello I, Bracaglia C, Strippoli R, De Benedetti F. Inhibition of natural killer (nk) cell cytotoxicity by interleukin-6: implications for the pathogenesis of macrophage activation syndrome. Pediatr Rheumatol. 2014;12 Suppl 1:56.

27. Yokota S, Itoh Y, Morio T, Sumitomo N, Daimaru K, Minota S. Macrophage activation syndrome in patients with systemic juvenile idiopathic arthritis under treatment with tocilizumab. J Rheumatol. 2015;42(4):712-22.

28. Ravelli A, Grom AA, Behrens EM, Cron RQ. Macrophage activation syndrome as part of systemic juvenile idiopathic arthritis: diagnosis, genetics, pathophysiology and treatment. Genes Immun. 2012;13(4):289-98.

29. Boom V, Anton J, Lahdenne P, Quartier P, Ravelli A, Wulffraat NM, et al. Evidence-based diagnosis and treatment of macrophage activation syndrome in systemic juvenile idiopathic arthritis. Pediatr Rheumatol Online J. 2015:13:55. Pubmed Central PMCID: PMC4669611.

30. Zeft A, Hollister R, LaFleur B, Sampath P, Soep J, McNally B, et al. Anakinra for systemic juvenile arthritis: the rocky mountain experience. J Clin Rheumatol. 2009;15(4):161-4

31. Nigrovic PA, Mannion M, Prince FH, Zeft A, Rabinovich CE, van Rossum MA, et al. Anakinra as first-line disease-modifying therapy in systemic juvenile idiopathic arthritis: report of forty-six patients from an international multicenter series. Arthritis Rheum. 2011;63(2):545-55.

32. Grom AA, llowite NT, Pascual V, Brunner HI, Martini A, Lovell D, et al. Rate and clinical presentation of macrophage activation syndrome in patients with systemic juvenile idiopathic arthritis treated with canakinumab. Arthritis Rheumatol. 2016;68(1):218-28.
33. Put K, Avau A, Brisse E, Mitera T, Put S, Proost P, et al. Cytokines in systemic juvenile idiopathic arthritis and haemophagocytic lymphohistiocytosis: tipping the balance between interleukin-18 and interferon-gamma. Rheumatology. 2015;54(8):1507-17.

34. Novick D, Kim S, Kaplanski G, Dinarello CA. Interleukin-18, more than a Th1 cytokine. Semin Immunol. 2013;25(6):439-48.

35. Jordan MB, Hildeman D, Kappler J, Marrack P. An animal model of hemophagocytic lymphohistiocytosis $(\mathrm{HLH}): \mathrm{CD} 8+\mathrm{T}$ cells and interferon gamma are essential for the disorder. Blood. 2004;104(3):735-43.

36. Krebs P, Crozat K, Popkin D, Oldstone MB, Beutler B. Disruption of MyD88 signaling suppresses hemophagocytic lymphohistiocytosis in mice. Blood. 2011;117(24):6582-8. Pubmed Central PMCID: PMC3123024.

37. Yanagimachi $M$, Naruto $T$, Miyamae $T$, Hara T, Kikuchi M, Hara R, et al. Association of IRF5 polymorphisms with susceptibility to macrophage activation syndrome in patients with juvenile idiopathic arthritis. Rheumatol. 2011;38(4):769-74.

38. Canna SW, de Jesus AA, Gouni S, Brooks SR, Marrero B, Liu Y, et al. An activating NLRC4 inflammasome mutation causes autoinflammation with recurrent macrophage activation syndrome. Nat Genet. 2014;46(10):1140-6. Pubmed Central PMCID: 4177369.

39. Romberg N, Al Moussawi K, Nelson-Williams C, Stiegler AL, Loring E, Choi M, et al. Mutation of NLRC4 causes a syndrome of enterocolitis and autoinflammation. Nat Genet. 2014;46(10):1135-9. Pubmed Central PMCID PMC4177367.

40. Strippoli R, Caiello I, De Benedetti F. Reaching the threshold: a multilayer pathogenesis of macrophage activation syndrome. J Rheumatol. 2013; 40(6):761-7.

41. Bracaglia C, de Graaf K, Pires Marafon D, Guilhot F, Ferlin W, Prencipe G, et al. Elevated circulating levels of interferon- $\gamma$ and interferon- $\gamma$ induced chemokines characterize patients with macrophage activation syndrome complicating systemic juvenile idiopathic arthritis. Ann Rheum Dis. 2017; 76(1):166-72.

42. Groom JR, Luster AD. CXCR3 ligands: redundant, collaborative and antagonistic functions. Immunol Cell Biol. 2011;89(2):207-15. Pubmed Central PMCID: 3863330

43. Behrens EM, Canna SW, Slade K, Rao S, Kreiger PA, Paessler M, et al. Repeated TLR9 stimulation results in macrophage activation syndrome-like disease in mice. J Clin Invest. 2011;121(6):2264-77. Pubmed Central PMCID: 3104738.

44. Buatois V, Chatel L, Cons L, Lory S, Richard F, Guilhot F, et al. Use of a mouse model to identify a blood biomarker for IFNgamma activity in pediatric secondary hemophagocytic lymphohistiocytosis. Transl Res. 2016; S1931-5244(16):30157-8.

45. Jordan ML F, Allen C, De Benedetti F, Grom AA, Ballabio M, Ferlin WG De Min C. A novel targeted approach to the treatment of hemophagocytic lymphohistiocytosis $(\mathrm{HLH})$ with an anti-interferon gamma (IFNy) monoclonal antibody (mAb), NI-0501: first results from a pilot phase 2 study in children with primary HLH. In: American society of hematology - 57th annual meeting \& exposition - LBA-3. 2015.

46. Gupta S, Weitzman S. Primary and secondary hemophagocytic lymphohistiocytosis: clinical features, pathogenesis and therapy. Expert Rev Clin Immunol. 2010;6(1):137-54.

47. Janka GE. Familial and acquired hemophagocytic lymphohistiocytosis. Annu Rev Med. 2012;63:233-46.

48. Xu XJ, Tang YM. Advances in studies on hemophagocytic lymphohistiocytosis: diagnosis and treatment. Zhonghua er ke za zhi Chinese J Pediatr. 2011:49(9):712-6. 